\title{
A Facility to Test Short Superconducting Accelerator Magnets at Fermilab
}

\author{
M.J. Lamm, C. Hess, D. Lewis, T. Jaffery, W. Kinney, J.P. Ozelis and J. Strait \\ Fermi National Accelerator Laboratory \\ P.O. Box 500, Batavia, Illinois 60510 \\ J. Butteris and A.D. McInturff \\ SSC Laboratory \\ 2550 Beckleymeade Ave., Dallas, Texas 75237 \\ K.J. Coulter \\ Argonne National Laboratory \\ 9700 South Cass Avenue, Argonne, Illinois 60439
}

October 1992

Presented at the 1992 Applied Superconductivity Conference, Chicago, Illinois, August 23-28, 1992 


\section{Disclaimer}

This report was prepared as an account of work sponsored by an agency of the United States Government. Neither the United States Government nor any agency thereof, nor any of their employees, makes any warranty, express or implied, or assumes any legal liability or responsibility for the accuracy, completeness, or usefulness of any information, apparatus, product, or process disclosed, or represents that its use would not infringe privately owned rights. Reference herein to any specific commercial product, process, or service by trade name, trademark, manufacturer, or otherwise, does not necessarily constitute or imply its endorsement, recommendation, or favoring by the United States Government or any agency thereof. The views and opinions of authors expressed herein do not necessarily state or reflect those of the United States Government or any agency thereof. 


\title{
A Facility to Test Short Superconducting Accelerator Magnets at Fermilab*
}

\author{
M. J. Lamm, J. Butteris ${ }^{\dagger}$, K. J. Coulter ${ }^{\dagger \dagger}$, C. Hess, D. Lewis, T. Jaffery, W Kinney, A. D. McInturff ${ }^{\dagger}$, J. P. Ozelis, and J. \\ Strait \\ Fermi National Accelerator Laboratory \\ Batavia, Il. 60510
}

\begin{abstract}
During the past four years the Superconducting Magnet R\&D facility at Fermilab (Lab 2) has successfully tested superconducting dipole, quadrupole, and correction coil magnets less than 2 meters in length for the SSC project and the Tevatron D0/B0 Low- $\beta$ Insertion. During this time several improvements have been made to the facility that have greatly enhanced its magnet testing capabilities. Among the upgrades have been a new rotating coil and data acquisition system for measuring magnetic fields, a controlled flow liquid helium transfer line using an electronically actuated cryo valve, and stand-alone systems for measuring $\mathrm{AC}$ loss and training low current Tevatron correction coil packages. A description of the Lab 2 facilities is presented.
\end{abstract}

\section{INTRODUCTION}

Fermilab has established two complementary facilities for testing superconducting magnets. The Magnet Test Facility (MTF) is equipped to test cryostated superconducting magnets up to $17 \mathrm{~m}$ in magnetic length. Non-cryostated superconducting magnets less than 2 meters in length can be tested at the Superconducting Magnet R\&D Laboratory (Lab 2). Tests are performed in vertical dewars of boiling liquid helium.

A major effort at both facilities in the past five years has been the R\&D and production testing of Tevatron Low- $\beta$ insertion magnets[1] and R\&D for the SSC model dipole program [2]. This paper describes the Lab 2 facility and the several upgrades which were developed to accomplish these successful test programs.

\section{CRYOGENIC SYSTEM}

Superconducting magnets are tested in three in-ground dewars as well as several smaller portable dewars. A simplified schematic of the cryogenic system is shown in Fig. 1. For clarity, only one dewar (dewar I), is shown. The helium and liquid nitrogen (LN2) vent/vacuum and supply portions of the system are similar for all test dewars.

Dewar I consists of two concentric stainless steel cylinders. The inner cylinder is $3.6 \mathrm{~m}$ in length, with a $45 \mathrm{~cm}$ inner diameter. The annulus between the two stainless steel cylinders contains a LN2 shield made of spiral wrapped copper tubing attached to a rolled copper sheet. The LN2

\footnotetext{
* Work Supported by the Department of Energy.

$\dagger$ Present Address: SSC Lab., Dallas, TX 75237.

†† Present Address: Argonne Nat. Lab., Argonne, Il 60439.

Manuscript received August 24, 1992
}

shield is wrapped with superinsulation. During cryogenic shield is evacuated. Above atmospheric dewar pressure test can be run by manually controlling a system of cryogenic ball valves. The dewar exhaust can be vented to atmosphere, connected to a system of Stokes vacuum pumps, or connected to a pressure building loop. The dewar can operate at pressures from 180 to 900 Torr which corresponds to boiling helium from $3.0 \mathrm{~K}$ to $4.4 \mathrm{~K}$.

The top plate of the dewar consists of a $2.54 \mathrm{~cm} \mathrm{G-10}$ plate. The magnets are suspended from this top plate with stainless steel threaded rods. Intrusions were made in the G-10 top plate to accommodate hermetically sealed instrumentation feed-throughs, an anticryostat, and vapor cooled power leads. Instrumentation wires fed through these ports include carbon and platinum thermometers, liquid level gages as well as magnet instrumentation such as voltage taps and strain gages. The anticryostat, used for room temperature probe insertion into the magnet bore, consists of concentric seamless stainless steel tubing. The annulus between the tubes is filled with superinsulation and evacuated. On some magnets, copper refrigerator tubing is wrapped around the magnet shell. During the initial stages of cooldown the tubing is connected in series with the dewar nitrogen shield LN2 line and used as a heat exchanger.

The liquid helium is delivered to the test dewar from $500 \mathrm{~L}$ vendor supply dewars via a three section transfer line. The first section, starting from the supply dewar, is a commercially made MVE flexible line. The additional sections were in-house designed and constructed. Section two is an

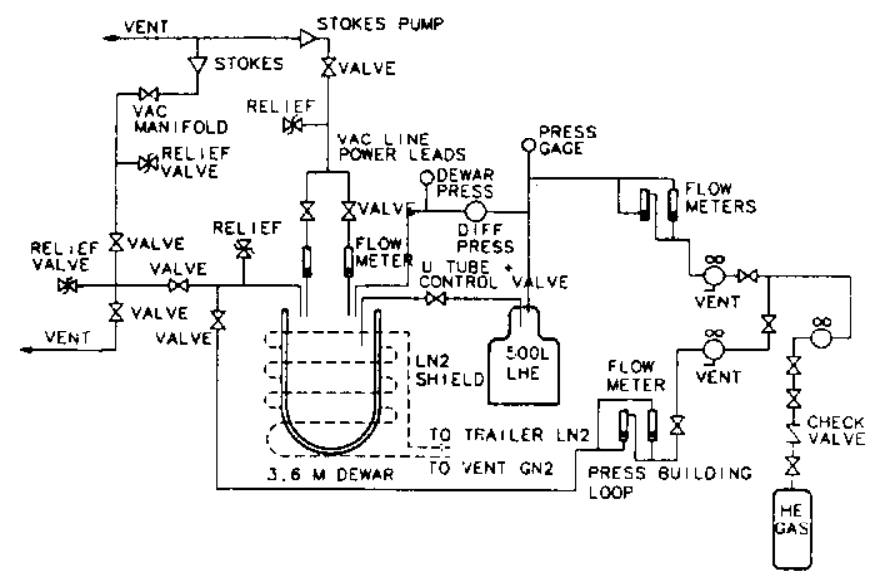

Fig. 1. Cryogenic System Block diagram. Only one of the three vertical dewars (dewar 1 ) is shown for clarity. 
inverted " $U$ " tube and contains a Cryolab CV9 globe valve with an equal percent flow plug option. This valve is located near the center of the line. The valve is operated by a modified Industrial Devices Corp. electrical actuator and uses a Schaevitz LVDT as a valve position indicator. Section three is a hard line " $U$ " tube that completes the transfer line run to the test dewar. All three sections are connected via bayonet style ends and use Huntington "Quick Couplings" for seals.

This three section transfer line has several noteworthy features. The electronically actuated globe valve can be automatically adjusted for continuous flow, thus minimizing pressure fluctuations associated with starting/stopping helium flow. The system is also very modular. The control system is discussed below. The three sections can be configured in different orientations. This allows more freedom in placing the supply dewar with respect to the test dewar.

\section{POWER SYSTEM}

A block diagram of the electrical power system is shown in Fig. 2. The system is normally configured with two Transrex (TR) power supplies in parallel giving a current capacity in excess of $10 \mathrm{kA}$. The Transrex current is controlled through a drive voltage signal supplied by a Fermilab designed CAMAC module. The current is read with a $10 \mu \mathrm{ohm} 12 \mathrm{kA}$ shunt. An I-dot coil is used as part of the quench detection system and recorded for off-line data analysis. The power system bus for each Transrex line consists of $7 \mathrm{~cm}$ outer diameter, $0.6 \mathrm{~cm}$ wall water cooled copper. The system ground is provided through a $1 \mathrm{ohm}$ and a $25 \mathrm{ohm}$ resistor placed in series. The voltage across the resistors is monitored through the data acquisition system in order to detect ground faults.

A "current dumping" circuit is used as a method of dissipating the magnet's stored energy which can be as high as $0.4 \mathrm{MJ}$. The dump system is activated through a dump SCR (SCR 11) which causes the $115 \mathrm{mF} 400$ volt capacitor to discharge. This results in a reverse-bias of the 10 element SCR run (SCR1-10), causing the current to flow through the 3 mohm resistor.

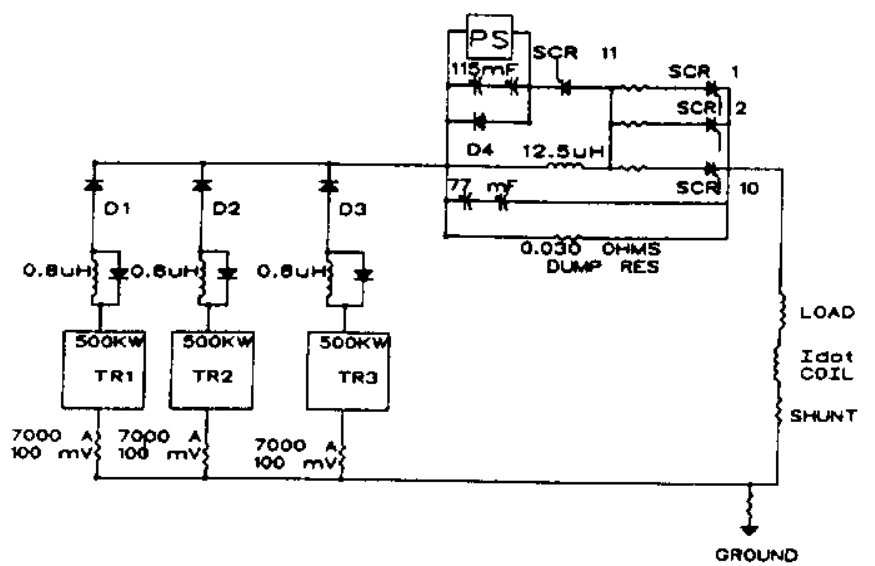

Fig. 2 Block diagram of electrical bus. During normal operation current flows through 10 parallel SCR run (SCR1-10) and $12.5 \mu \mathrm{H}$ inductor.
Interlocks have been installed to prevent the power supply from operating during certain unsafe conditions. When an interlock condition occurs during current excitation, the power supply is phased to zero current and the dump capacitor is fired. Interlocks include temperature sensors on the bus cooling water and dump resistor, magnetic field sensors that detect high current through the dump resistor, water flow sensors in the cooling water system and door interlocks to enclosures that contain exposed electrical bus.

Additionally, there are a system of quench detection circuits that detect a non-normal operating condition in the magnet or power system. These circuits have been discussed in detail elsewhere [3]. Non-normal operating conditions fall into two categories. One is associated with a detection of a resistive voltage growth in the superconducting magnet. In this case the power supply is phased off and the dump circuit is enabled. In the other, a system fault is detected such as a ground fault or the vapor cooled power leads are overheated. In this case, the large voltage across the magnet associated with the enabled dump circuit is not desirable, so the power supplies are ramped to zero current through a Fermilab designed CAMAC ramp down module.

\section{DATA ACQUISITION /SYSTEM CONTROL}

\section{A. Overview}

Much of the instrumentation found on magnets tested at Lab 2 and MTF are identical. In some cases a magnet was first tested in Lab 2 in a vertical dewar, and later tested in MTF after being placed in a cryostat. Thus an effort was made to keep the data acquisition hardware and software as compatible as possible.

The power supply control and magnetic instrumentation readout system is an example of this compatibility. The drive signal for the Transrex power supply system, as well as the readout for most magnet instrumentation such as voltage taps, was controlled by a DEC MicroVax II. This system has been described in detail elsewhere.[3,4]. The key features of the MicroVax system are LeCroy 8212A CAMAC ADC moduies to read isolation amp protected voltage tap signals, and IEEE bus through CAMAC readout of Hewlett-Packard HP3457a DVMs to read voltages from strain gage, cryogenic instrumentation and the magnet shunt.

Several new measurement and control lasks required for Lab 2 tests were accomplished through stand alone PC or UNIX based systems. This approach allows maximum programming and experiment configuration flexibility.

\section{B. Liquid Helium Transfer}

Cryogenic system parameters such as supply and test dewar pressure, test dewar liquid level, vapor cooled power lead flow and the position of the transfer line cryogenic globe valve (LVDT) are monitored by a stand-alone AST 386 computer based system nunning the Keithley/ASYST ViewDac software package. The computer is equipped with MetraByte DAS8 A/D boards, a DDA06 D/A board and an IEEE 488 interface. 
ViewDac uses the liquid level, globe valve LVDT as well as operator input PID parameters to automatically control the position of the globe valve. This eliminates the need for continual operator intervention, and provides for a stable flow of helium. The cryogenic data can be displayed for on-line system diagnostics or can be uploaded to the MicroVax II for further analysis.

\section{AC loss system}

The energy loss in a superconducting magnet during a high current cycle is measured by using a stand-alone PC-based data acquisition system. The magnet current is executed by the MicroVax controlled Transrex power system. The PC reads the magnet current and voltage from two HP3457a DVMs through a GPIB interface. The readings are synchronized by a GPIB controlled wave form generator. The time integrated product of current and voltage over a complete cycle gives the energy loss/cycle. A detailed description of the AC loss measurement system as well as results from several SSC magnet tests is presented elsewhere [5].

\section{Magnetic Measurement System}

\section{Magnetic Probes}

The magnetic field measuring coils are made from formvared wire laid onto the surface of grooved G-10 cylinders. The coils have been built using the Morgan [6] and tangential coil geometry. The length of the probe is chosen so that the probe can be placed well within the magnet body. Typical length is $25-50 \mathrm{~cm}$.

The G-10 coil form is attached to a hollow G-10 shaft. The shaft sections are joined together by journals made of G-10 and Micarta. For probe rotational stability, bearings made of DuPont Delrin ${ }^{\circledR} \mathrm{AF}$ are placed on the surface of these journals that fit snugly with the inner wall of the anticryostat. The probe shaft extends beyond the mouth of the anticryostat where it is connected though a mechanical coupling to a gear shaft. The gear shaft assembly can be moved vertically approximately 2 meters. The vertical movement is performed by a PC controlled stepping motor. The length of the probe shaft is custom fit to the particular magnet under test so that the probe can traverse the length of the magnet with the available motion of the drive shaft. There is also a rotational encoder module located in series with the gear shaft. The probe is typically rotated at an angular speed of $6 \mathrm{~Hz}$. The speed is limited by the mechanical stability of the probe shaft system. The coil leads are twisted and led through the center of this shaft. The signal wires are then attached to mercury wetted slip rings. The magnetic field coil wires and encoder signals are then fed into the signal processing system.

\section{Signal Processing System}

A system was designed to convert the Morgan and tangential coil voltages and encoder pulses into magnetic field harmonics. A V/F converter based system was chosen over an $A D C$ based system because it is less sensitive to probe speed variations. This is because the $\mathrm{V} / \mathrm{F}$ converter effectively time integrates the signal voltage. After normalizing for geometric factors and amplifier gains, the scaler count of the $V / F$ converter output as a function of encoder pulse yields the magnetic flux as a function of rotation angle. This distribution can be reduced to harmonic coefficients by use of a Fourier transform analysis.

A block diagram of the system is shown in Fig 3. The data acquisition computer is a Concurrent 6400: a UNIX based real time computer with a VME bus. The Concurrent 6400 also has a GPIB interface through which an HP3475A DVM reads the shunt current. It also has an ethernet link to the MicroVax which allows for easy uploading of data for off line analysis. The Concurrent acts as a user interface to the data acquisition hardware, which is located in a 21 slot $6 \mathrm{U}$ VME crate attached to the Concurrent extended VME bus.

The co-processor for the system is a 68000 based Force Computers IPIO board. The IPIO board is responsible for transferring the user instructions to the system, and collecting scaler readings as a function of encoder pulse count.

Besides the IPIO board and the VME crate-extender card, the system includes several Lab 2-designed boards resident on the VME crate: a coil signal gain/attenuator card, V/F converter scaler card, encoder signal generator/simulator and a IPIO to L2bus converter. For these card locations, the backplane of the VME crate was modified. The standard VME bus was replaced by a digital (called L2bus) and analog bus. In order to reduce the noise from the digital signals, the analog bus was physically separated from the digital bus and was powered by its own high quality linear power supply.

The gain/attenuator card function is to take the differential coil signal, and turn it into a single ended signal whose amplitude is amplified/attenuated to match the range of the $\mathrm{V} / \mathrm{F}$ converter. The gain setting can be set manually through the Concurrent interface program or can be set automatically. The resulting signal is placed on the analog bus to the corresponding $\mathrm{V} / \mathrm{F}$ board.

The V/F board contains a bipolar Analog Devices AD652 $\mathrm{V} / \mathrm{F}$ converter ( $1 \mathrm{MHz}$ corresponds to +5 volts). The time base for the $\mathrm{V} / \mathrm{F}$ converter is provided by a $2 \mathrm{MHz}$ quartz clock. The resultant TTL logic pulses from the V/F converter

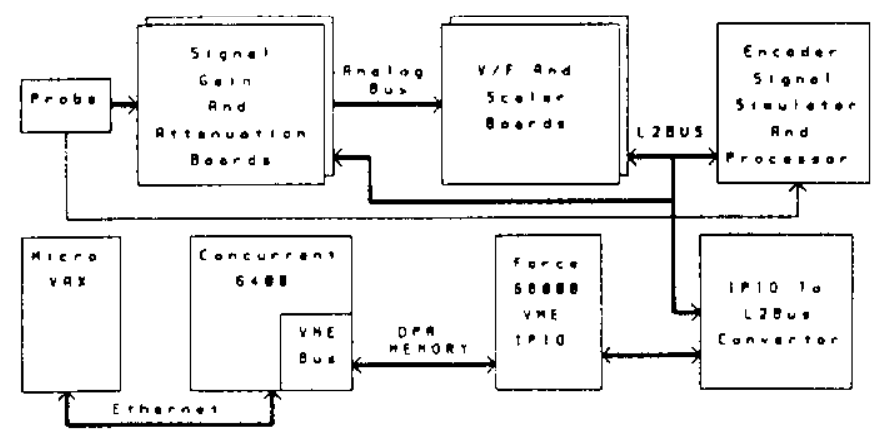

Fig. 3 Block diagram of magnetic measurement system. 
are sent to 32 bit scalers. One scaler board is dedicated to reading pulses from the quartz timing clock. This information, along with zero volt calibration data for each V/F channel is used to remove the $0.5 \mathrm{MHz}$ zero volt offset from the bipolar V/F converter.

The scaler reads are triggered by the prescaled encoder signal from the encoder signal generator board. The readings are transferred via L2 bus to the IPIO to L2bus converter board, which in turn is read by the IPIO unidirectional digital input lines. The data are then stored in a dual port RAM (DPR) which is directly accessible to the Concurrent.

\section{E. Tevatron Correction Coil Test System}

The Fermilab Low $\beta$ Insertion project requires the construction of over 60 magnets, including 30 "spool" piece elements. Most spool pieces consist of a high gradient quadruple and one correction coil package. The correction coil package consists of two or three concentric free wound potted coils. For example, dipole, sextupole and quadruple windings are packaged together. Typical operating requirements for a correction coil winding is $0-50$ amps in either polarity independent of the other correction coil winding current/polarity. The correction coils are required to demonstrate that they can operate under these conditions without quenching prior to placing these magnets into cryostats. Typically, a correction coil package would undergo 10 training quenches to achieve this.

These tests needed to proceed in parallel with the tests of other high gradient quadrupoles as well as the SSC model dipole program, with a minimum of interruption to the MicroVax data acquisition system. Furthermore, the power requirements (three bipolar independent 200 amp power supplies) are incompatible with the high current power system.

The solution is a stand-alone PC-based data acquisition system. The system diagram is shown in Fig. 4. The system consists of an AST Premium 286 computer, three Sorenson

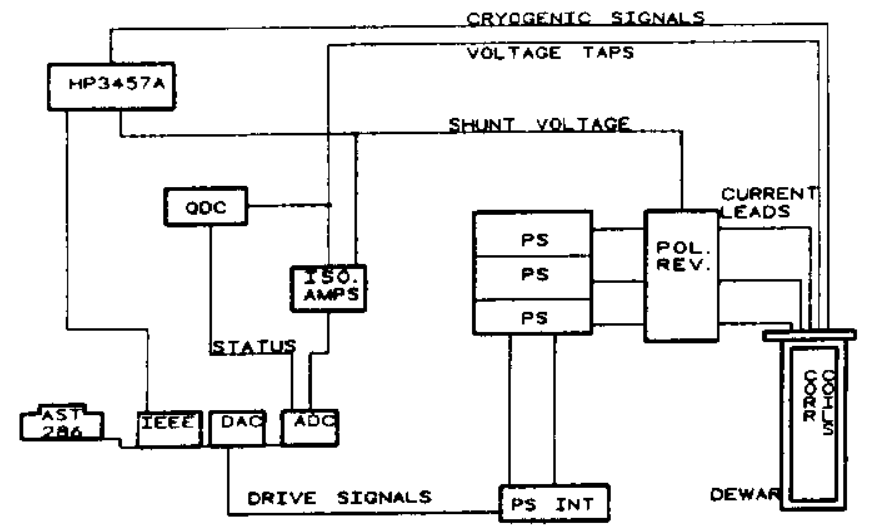

Fig. 4 Block Diagram of Tevatron Correction Coil data

\footnotetext{
acquisition system.
}

DC32-155T power supplies (30 volt, 200 amps) operated in a current regulated mode (PS), an SCR based polarity reversing switch (Pol. Rev.), and a quench detection circuit (QDC).

The AST computer contains three boards, a MetraByte DAS8 A/D, MetraByte DDA06 D/A and a IEEE interface board. The D/A produces a drive voltage signal used to remotely control each power supply. The A/D records magnet voltages, typically at $0.2 \mathrm{KHz}$. The IEEE interface is connected to a multi channel HP3457a DVM which in tum reads voltages from dewar thermometry and pressure sensors as well as the shunt currents.

Once a quench is detected through a coil QDC, a status signal is sent to the Power supply interface module(PS Init). The module in turn drives the ramped drive voltage to zero. It also instructs the PC to take ADC data for a set period of time so that the post quench voltage behavior can be recorded. The data are written to disk, thus maintaining a complete quench history of the correction coil package. The data also could be uploaded to the MicroVax for off-line data reduction using the computer serial port.

\section{CONCLUSIONS}

This paper describes the Superconducting Magnet R\&D Facility at Fermilab (Lab 2). Included are several newly designed PC and UNIX based data acquisition systems. These systems have been successfully used for a wide range of magnet test applications.

\section{ACKNOWLEDGMENT}

The authors wish to acknowledge the Lab 2 staff for their contributions to the development of this facility.

\section{REFERENCES}

[1] S. Gourlay et al., "Quench Performance of Superconducting Quadrupole Magnets for the New Fermilab Low Beta Insertion.," Proc. of the 1991 IEEE Part. Accel. Conf, San Francisco, Ca, Vol 4.

[2] T. Jaffery et al., "Test Results of Post-ASST Design Fermilab Built 1.5 Meter, SSC Dipole Magnets,"paper LOA-6, this conference.

[3] J. Strait, et al., "Fermilab R\&D Test Facility for SSC Magnets," Supercollider I, 1989.

[4] K. McGuire, et al., "Cryogenic Instrumentation of an SSC Magnet Test Stand," Adv. Cryo. Engr. 33, 1988.

[5] J. P. Ozelis, et al., "AC Loss Measurements of Model and Full Size $50 \mathrm{~mm}$ SSC Collider Dipole Magnets at Fermilab," paper LOA-9, this conference.

[6] G. H. Morgan. "Stationary coil for Measuring the Harmonics in Pulsed Transport Magnets," Proc. Inter. Conf. on Magnet Technol., 1972. 